\title{
Frontières
}

\section{Deuil et spiritualité chrétienne}

Un parcours d'espoir avec Lytta Basset

\section{Denise Badeau}

Volume 18, numéro 2, printemps 2006

La mort dans tous ses états

URI : https://id.erudit.org/iderudit/1073225ar

DOI : https://doi.org/10.7202/1073225ar

Aller au sommaire du numéro

Éditeur(s)

Université du Québec à Montréal

ISSN

1180-3479 (imprimé)

1916-0976 (numérique)

Découvrir la revue

Citer ce document

Badeau, D. (2006). Deuil et spiritualité chrétienne : un parcours d'espoir avec

Lytta Basset. Frontières, 18(2), 69-73. https://doi.org/10.7202/1073225ar d'utilisation que vous pouvez consulter en ligne.

https://apropos.erudit.org/fr/usagers/politique-dutilisation/ 


\section{$\begin{array}{llllllllll}P & O & \text { I } & N & T & D & E & V & U & E\end{array}$}

\section{DEUIL \\ ET SPIRITUALITÉ CHRÉTIENNE Un parcours d'espoir avec Lytta Basset}

Denise Badeau, Ph.D., professeure associée, Département de sexologie, UQAM.

Selon Lytta Basset ${ }^{1}$, théologienne et philosophe, il importe, en situation de deuil, de rester ou de redevenir disponible au Souffle qui continue de nous régénérer et de nous animer. Lytta Basset aborde la spiritualité et le deuil en se référant au Nouveau Testament. Elle cite une définition de la mort puisée dans le livre de Kübler-Ross (2005, p. 59): La mort est un nouveau soleil, qui dit ceci: "passage dans un nouvel état de conscience dans lequel on continue de sentir, de voir, d'entendre, de comprendre, de rire... où l'on a la possibilité de continuer à grandir». Et aussitôt un premier lien est ménagé entre cette définition et le verset 48 de Mt 5 : ils avaient une impression "d'intégralité » et «vous serez parfait, accompli, comme votre Père céleste est parfait (complètement fait), accompli» (traduction de Lytta Basset).

Selon la théologienne, la mort s'inscrit dans ce trajet pour aller plus loin. Toute l'existence humaine est orientée en vue de «afin que». Souvent, dit-elle, on croit que la vie n'a pas de sens parce qu'on a perdu son orientation, son "orient».

Le deuil serait, selon Marie-Frédérique Bacqué et Michel Hanus (2005), un «état affectueux, douloureux provoqué par la mort d'un être aimé. C'est aussi la période de douleur et de chagrin qui suit cette disparition». Freud (1917) le définit comme la réaction habituelle à la perte d'une personne aimée ou d'une abstraction mise à sa place: la patrie, un idéal, la liberté, etc. Pour Christophe Fauré, le deuil est un processus normal et incontournable se déclenchant après chaque perte: ressenti physique, état psychique, événement social et relationnel. Les différentes étapes du deuil, telles que pressenties par E. Kübler-Ross, sont une référence incontournable.

Quant à la spiritualité, elle appartient, selon Lytta Basset, à tout être du seul fait de son existence. Elle concerne sa relation aux valeurs qui le transcendent quel que soit le nom qu'il leur donne (voir prologue, Jean 1,1-18). La spiritualité est l'ouverture à l'expérience du Christ dans la vie quotidienne, elle est faite d'accueil, d'approfondissement. Cette dimension est rarement prise en compte parce qu'elle n'a pas de statut, pas de légitimité dans ce monde. La spiritualité chrétienne vit de l'esprit du Christ, elle est le cheminement terrestre en quête de cette vérité de la Parole qui nous conduit vers la vie. Voie-vérité-vie. Chaque parcours de l'Esprit, quel qu'il soit, et le langage dans lequel il est communiqué expriment une façon personnelle de recevoir l'Évangile, le Souffle. L'accompa- gnement spirituel serait l'accueil de la personne dans sa souffrance, dans sa réalité, sa vérité du moment. Chacun essaie d'avancer avec ses propres pertes. Accueillir l'expérience de l'autre et que l'autre accueille mon expérience, c'est spirituel. Les mots, on les trouve selon la qualité de la relation. Le bonheur que Lytta Basset appelle joie «imprenable» inclut, englobe tout ce qui nous paraît négatif.

La religion est la réponse que l'humanité a tenté de donner à ces questions à travers un ensemble de pratiques et de croyances. Le logos (parole) éclaire tout être humain venant en ce monde. Dans la religion chrétienne, Dieu est transcendant, Il s'est révélé par Jésus de Nazareth. L'Esprit, lui, souffle où Il veut, quand Il veut, esprit de liberté et de vérité.

\section{LES RÉSISTANCES}

\section{AU TRAVAIL DE DEUIL}

On connaît très peu de lieux où l'on parle de la mort qui va venir. Il existe un tabou sur la mort et la spiritualité. On nourrit le mythe de l'éternelle jeunesse. La mort, affirme Lytta Basset, est une réalité qui a une dimension spirituelle énorme. Devant la mort d'autrui et la perspective de ma propre mort, je peux être terrifiée. Ce peut même être la trace d'une mort que j'ai déjà vécue. Il y a un ou des événements de vie qui m'ont tuée, qui ont détruit ma capacité 
d'être en lien et dont les indices seraient: angoisse, peur de l'abandon, destruction de la confiance; «je ne serai plus en lien avec personne», peur de l'anéantissement (ne plus exister pour personne), peur d'être complètement oublié, perdu. Or, ces événements ne sont pas toujours mis au jour. Il est donc important de rechercher la vérité sur sa propre histoire et sur les pertes qui ont provoqué ces angoisses.

Certains auteurs parlent de l'existence au négatif concrétisée par tout ce qui fait mal (échec, abandon, trahison, déception) et qui menace notre désir de vivre. Paul Ricoeur, quant à lui (r.i.) parle de «la vivante nonnécessité d'exister». L'existence au négatif me fait croire que je ne suis pas si nécessaire que ça, il s'agit d'une fêlure secrète, un nonmoi. Ce non-moi fait partie de moi et me fait comprendre que Dieu me réserve plus que ça, il m'appelle à «faire avec » ces blessures invisibles. Nous savons que «toutes choses contribuent (travaillent) au bien de ceux qui aiment Dieu », de ceux qu'Il a appelés selon son plan. (Rom. 8, 28.) Dans la vie spirituelle, il y a parfois des passages à vide: «Dieu délivrera le malheureux à travers son malheur et il découvrira son oreille à travers l'oppression » (Job 36, 15).

La première perte essentielle se produit à la naissance. Par la suite, chaque fois qu'il y a un passage, il y a acquisition du «je» perte. Je ne peux pas accueillir cette expérience à la place de l'autre, je ne peux pas faire l'apprentissage de la solitude à sa place.

Celui qui vient à moi doit me préférer

à son père, sa mère, sa femme, ses

enfants, ses frères, ses sœurs, et même

à sa propre personne. Sinon, il ne peut

être mon disciple. Celui qui ne porte

pas sa croix pour me suivre ne peut

pas être mon disciple. (Lc 14, 26-27)

«Puis il dit à tous: "si quelqu'un veut venir avec moi, qu'il cesse de penser à luimême, qu'il porte sa croix chaque jour et qu'il me suive"»(Lc 9, 23). Il est important de se mettre debout avec ce qui est douloureux pour suivre Jésus; se «mettre debout» avec les ressources du moment, avec sa croix; lever sa croix chaque jour; s'arracher à la fusion, personne ne peut le faire à la place de quelqu'un d'autre. Il n'y a pas d'âge pour faire l'apprentissage de la solitude du «je», pas d'âge pour accueillir le «non-moi» en moi. Ne pas minimiser la perte; bien souvent, on étouffe la douleur de la perte par crainte de faire rire de soi. Pourquoi avons-nous honte quand nous avons mal? Parce que nous nous sentons amoindris... «Oui, je vous le déclare, c'est la vérité: un grain de blé reste un seul grain s'il ne tombe pas en terre et ne meurt pas. $[\ldots] »(J n$ 12, 24). De fait, quitter ce qui nous est familier représente déjà la mort, et l'angoisse n'est jamais absurde, elle cache quelque chose.

Dans la place laissée libre par la perte, il faut d'abord vivre l'arrachement, il faut qu'un vide se produise; vide, nuit obscure, qu'il faut renoncer à combler soi-même. La Bible parle de l'extraordinaire fragilité de l'humain en même temps que de son infinie valeur. Pour aller vers l'acceptation, il faut passer par l'épreuve de la réalité. Mort, mur.

Le deuil ne survient jamais pour la première fois, l'être humain est très tôt confronté au manque qui est fondateur de la vie psychologique. L'aptitude au deuil se met en place dès le début de l'existence, dès les premières relations avec les autres et va suivre le développement de chacun, aidant à faire face non seulement à ses pertes et à ses deuils mais aussi à ses limites, à ses déceptions, à ses échecs.

\section{L'INTÉGRATION DE L' «IN-INTÉGRABLE » ET LA RE-DÉFINITION DE L'IMAGE DE SOI}

Que se passe-t-il quand on refuse un sentiment de perte? De l'impuissance, une image amoindrie de soi-même, l'impression de ne pas exister vraiment, de souffrir sans les autres, l'aveu de la rupture du lien avec les autres. Personne ne peut mesurer l'amoindrissement de mon être. Perte et solitude vont de pair, on ne peut faire l'économie de la solitude. Pour intégrer l'in-intégrable, je dois aller au fond de cette solitude où je vais trouver une capacité de me détacher et retrouver la liberté par rapport à ce que j'ai perdu. Je me détache de ceux ou de celles dont ma vie dépendait; se déconstruit ainsi mon fantasme de perfection. L'isolement m'enferme dans la mort, il est mortifère pour moi et pour l'entourage. De cette solitude radicale peut surgir un processus de vie: «je me prends dans les bras», je deviens mon propre parent. On ne peut accéder à une communion vivante sans passer par la solitude. Il faut relire la parabole du fils prodigue (voir Lc 15, 11-32): dans ce récit, chacun est dans le «souffrir sans»: le père sans son fils, le fils sans son père, le fils sans le nécessaire pour survivre, le frère aîné sans la reconnaissance du père dans la fête...

La perte est d'abord et avant tout une question d'identité: Je ne suis plus la femme de... le parent de... le fils de... Perte d'amour, d'amitié, de confiance. Quand vous serez déstabilisé par l'absurde, moi je vous supplie de croire que moi je suis. "Moi je suis», revient sept fois dans l'Évangile de Jean. Pour arriver à redéfinir son image, l'endeuillé doit se blottir dans ce «moi je suis».

\section{LES INDICES D'UNE FORCE DE RÉSURRECTION}

Mettre hors de soi ce qui étouffe, avec des mots, par des larmes, par des cris: « Vois, je te propose aujourd'hui vie et bonheur, mort et malheur» (Dt 30,15). Tu vivras, tu choisiras ta vie. En faisant ce choix d'opter pour la vie, il est bénéfique pour l'endeuillé-e et pour son entourage de ne pas rester otage de la personne décédée. Quand survient la mort d'un enfant, les autres enfants doivent sentir que les parents aiment encore la vie, qu'ils optent pour la vie, qu'ils ne restent pas otage de la mort, du mort ou de la morte. Entretenir la vie est le premier pas dans l'expérience de la résurrection.

Souvenons-nous de ce que la personne décédée a dit: la relecture de l'expérience fait partie du rite de séparation qui doit être accompli. La vie continue - elle ou il est bien là où il, elle est. Souvent l'endeuillé est séquestré par le "comme avant», il reste enfermé dans les images et la représentation de comme c'était avant; il essaiera, par exemple, d'atténuer la perte en remplaçant un enfant par un autre. Le vrai vivant, c'est quelqu'un qui vibre à mon chagrin au point d'en être pris aux entrailles; quelqu'un qui me dit une parole qui met fin à mes larmes, qui m'apaise. Jésus toucha la civière:

Et il arriva, le jour suivant, que Jésus alla dans une ville appelée Naïn, et faisaient route avec lui ses disciples et une foule nombreuse. Quand il fut proche de la porte de la ville, voici qu'on emportait un mort, fils unique de sa mère, qui était veuve, et une foule considérable de la ville était avec elle. En la voyant, le Seigneur fut pris aux entrailles pour elle. Il lui dit: « $\mathrm{Ne}$ pleure pas!» Et s'approchant, il toucha la civière, les porteurs s'arrêtèrent et il dit: "Jeune homme, je te dis, réveilletoi!» Alors le mort se dressa sur son séant et commença à parler. Et il le donna à sa mère. Une crainte les saisit tous, et ils glorifiaient Dieu en disant: «Un prophète, un grand, s'est réveillé/levé parmi nous» et «Dieu a visité son peuple». Et cette parole sortit à son sujet dans la Judée entière et dans toute la région. (Lc 7, 11-17; traduit par Lytta Basset.)

\section{LE PARDON À SOI-MÊME}

La culpabilité fait partie du deuil: «j'aurais dû, j'aurais pu sauver la personne». «J'aurais dû » fait croire à «j'aurais pu». Je n'en ai pas fait assez... ce qui peut aller jusqu'à l'«infini». Ça demande tout un travail pour se tenir face à l'inexplicable: je laisse tomber la culpabilité, je me trouve dans la souffrance à l'état pur, crucifié-e par la mort de quelqu'un à l'instar de Jésus. J'ai mal d'être coupable et suis par le fait même 


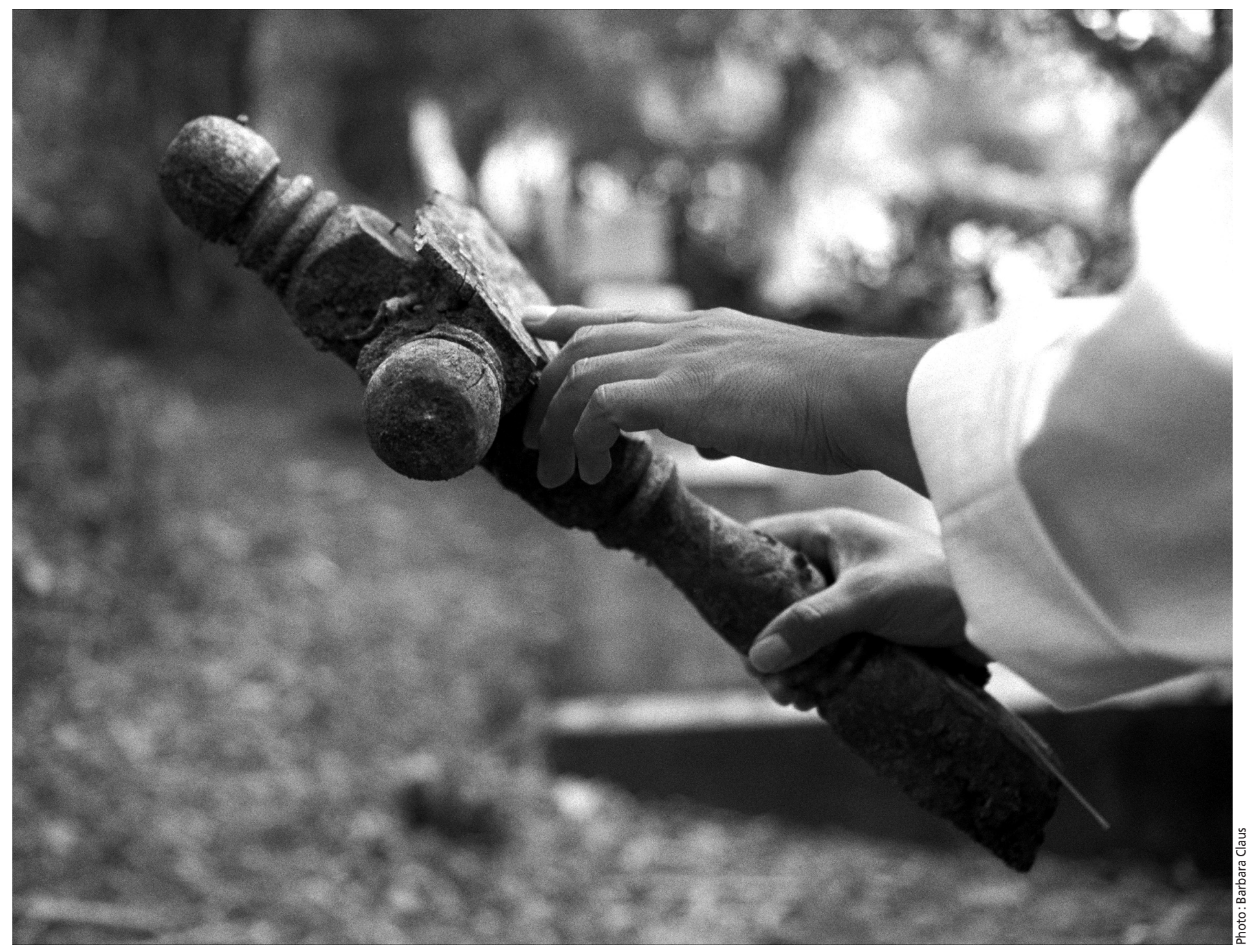

moins centré sur la disparition de l'autre, la culpabilité redonne une espèce de pouvoir face à la disparition mais paralyse si on s'y enferme. Distinguer la culpabilité qui se dissout de la culpabilité qui persiste. Reconnaître sa culpabilité dans les limites de ce qu'il était possible de faire. Se re-connaître tel qu'on est afin d'avoir accès à ce difficile apprentissage qui consiste à se pardonner soi-même en dépit de ce qu'on est et de ce qu'on aurait pu faire.

\section{SYMBOLISME DU TOMBEAU VIDE}

Le premier jour après le sabbat, Marie de Magdala vient vers le tombeau à l'aube, quand il fait encore sombre, et elle voit la pierre enlevée hors du tombeau. Alors elle court et va vers Simon Pierre et vers l'autre disciple, celui que Jésus aimait. Et elle leur dit: "Ils ont enlevé mon Seigneur hors du tombeau et nous ne savons pas où ils l'ont mis.» Ils sortirent donc, Pierre et l'autre disciple, et ils allèrent vers le tombeau, et les deux coururent ensemble. Et l'autre disciple courut devant plus vite que Pierre et arriva le premier dans le tombeau et s'étant baissé de côté, il voit les bandelettes posées là; pourtant il n'alla pas dedans. Arrive alors aussi Simon Pierre qui le suivait et il alla dedans, dans le tombeau. Et il contemple les bandelettes posées là et le linge fin qui était sur sa tête, non pas déposé avec les bandelettes mais roulé à part dans un endroit. Alors donc entra dedans, aussi, l'autre disciple venu le premier dans le tombeau, et il vit et il crut. En effet, ils n'avaient pas encore compris l'Écriture - qu'il lui fallait se lever d'entre les morts. Alors les disciples retournèrent chez eux.

(Jn 20, 1-10; traduction Lytta Basset, notre soulignement.)

Séquestré, chacun porte un tombeau scellé en dedans. On peut s'enfermer dans ce tombeau parce qu'il n'y a pas d'accueil. Marie vient dans le tombeau de son grand amour crucifié, vient s'approcher de l'horreur qu'elle a vécue, de l'horreur logée en elle. Le «nous» du texte peut représenter la Marie de Magdala souffrante et la Marie ressuscitée naissante. Elle voit son tombeau ouvert, elle voit son propre corps enlevé du tombeau, la pierre roulée devant son propre tombeau. Le Christ ou Jésus n'utilise jamais de «bulldozers », jamais de rouleaux compresseurs. Il laisse la liberté d'ouvrir sa porte ou non - cette porte qui ne s'ouvre que de l'intérieur. Marie a peur de l'anéantissement, de la «non-prise-en compte» de ce qu'elle a vécu.

Marie de Magdala se jette dans la relation, ne vérifie rien, ne sait pas, court vers les autres, vers la vie relationnelle. Vite, aussitôt, sur le champ! Urgence de la relation. Quand il s'agit de choses essentielles, il y a toujours urgence. Toujours de l'urgence quand il y a problème relationnel. C'est au moment où elle «court» vers les autres que le «nous» surgit. La personne ressuscitée: l'autre Marie.

Où es-tu toi que j'ai perdu? Jésus vient chercher l'autre en moi pour m'amener dans un espace de non-savoir. Jésus ne se 
réduit pas à un corps mort. Il faut entrer dans le tombeau pour entrer dans la résurrection. Tu entres ou tu n'entres pas ( 7 fois «tombeau» dans ce texte de Jean; 7, chiffre de la complétude). Autant de fois que nécessaire, je dois entrer dans le tombeau... jusqu'à l'achèvement du processus. Se tenir dans ce lieu de mort où il viendra me chercher. Tiens-toi en enfer et ne désespère pas. Quand je me tiens dans le tombeau, j'ai à aller au bout de ce temps où Jésus viendra chercher les vivants et les morts. Ils (Marie de Magdala, Simon Pierre et l'autre disciple) «courent». Toute une part de Pierre impétueuse et passionnée «court», Pierre aspire à démarrer sur de nouvelles bases. Il n'arrive pas à entrer dans le vide du tombeau, dans le néant de sa propre mort. Celui qui a renié Jésus va dans le tombeau vide de ses propres morts et constate que c'est vide (perte de tout repère). Le vivant est passé par là: les bandelettes, le linge fin en témoignent. L'autre disciple, l'autre Pierre tire en avant, c'est lui qui voit et croit (faire confiance). Il va dans le vide de son propre tombeau, va de l'avant. "Celui que Jésus aimait»: la partie de Pierre que Jésus aimait, la partie capable d'investir dans le lien, c'est la partie de lui qui tire en avant (Jn 21, 11). «Marie se tenait près du tombeau, dehors et pleurait.Tandis qu'elle pleurait, elle se baissa pour regarder dans le tombeau.» Marie fera la même démarche que Pierre, elle aura les mêmes repères.

Marcher sur le chemin de la résurrection, c'est se tourner (convertir) vers d'autres liens. À la recherche de ce qui était perdu: «Car le Fils de l'homme est venu sauver ceux qui étaient perdus» (Mt 18, 11). Chercher la part perdue dont nous ne connaissons pas l'ampleur, la part de régression. Le besoin de récupérer la personne perdue est une démarche très intense dans les premières semaines suivant la mort. Nous ne pouvons nous séparer de l'être perdu qu'après l'avoir recherché de toutes nos forces en vain. Pour que Lui, le vivant, puisse venir le chercher. Si vous voyez la main de Dieu, ça vient vous chercher.

\section{LA PÉRENNITÉ DU LIEN}

Lytta Basset interroge, dans la perte, le devenir du lien. Le meilleur antidote de la perte, c'est de traverser la perte de l'amour. Qu'est-ce que j'ai fait de mes relations avec l'autre et l'Autre? Comment suis-je en lien avec autrui décevant, perdu, mort, pour reprendre pied dans la vie? Comment parvenir à sentir, malgré tout, la consistance de l'autre?

Si c'est à cause de ta main ou de ton pied que tu tombes dans le péché, coupe-les et jette-les loin de toi; il vaut mieux pour toi entrer dans la vraie vie

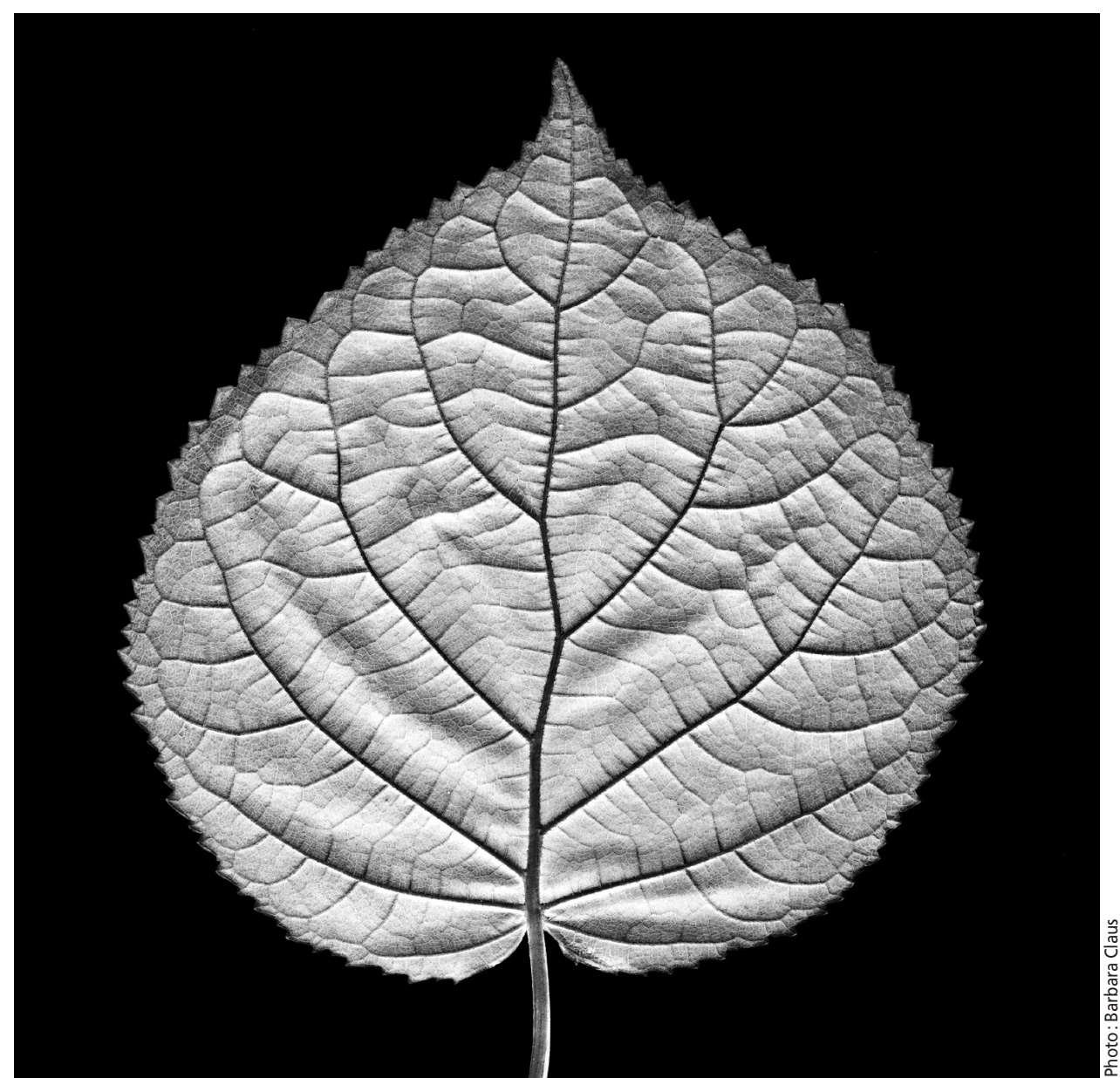

avec une seule main ou un seul pied que de garder les deux mains et les deux pieds et d'être jeté dans le feu éternel. Et si c'est à cause de ton œil que tu tombes dans le péché, arrachele et jette-le loin de toi; il vaut mieux pour toi entrer dans la vraie vie avec un seul œil que de garder les deux yeux et d'être jeté dans le feu de l'enfer. (Mt 18, 8-9)

Mieux vaut que tu... Je suis comme mutilée. La relation est possible parce que j'accepte mes cicatrices sinon je «n'entre pas dans la vie». Quelqu'un qui n'a jamais fait le travail d'acceptation de la perte va vivre en enfer... dépendant de... Dans le premier temps du deuil, on est comme mort. Il importe de savoir où lui ou elle se trouve en connaître le «lieu» nous permettrait de considérer l'autre comme encore vivant et de pouvoir l'aimer à nouveau. L'endeuillé espère un signe que l'autre existe à nouveau mais le devenir du lien dépend du devenir de l'identité de l'endeuillé. Je ne peux plus être en relation comme je l'étais auparavant. Je me déplace pour permettre au lien de se constituer à nouveau. "Je vous le dis déjà maintenant, avant que la chose arrive, afin que lorsqu'elle arrivera vous croyiez que "je suis qui je suis" »... (Jn 13, v 19).
Quelqu'un dont la confiance a été détruite pourra détruire la confiance de quelqu'un d'autre, très souvent de ses propres enfants. C'est comme si la partie la plus vivante de lui a été perdue. Il est donc souhaitable de revivre les affects en les partageant avec quelqu'un de compatissant qui va écouter, et ce, pour ne pas encombrer les autres, ses proches. "Demeurez en moi et moi en vous.» L'inclusion est utilisée à plusieurs reprises dans les Écritures: "Mes larmes sont à toi et tes larmes sont à moi. » Jésus nous enseigne comment traverser la perte.

Le passage où Jésus dit: «mon âme est triste à mourir» traduit bien le besoin de relations: "veillez et priez». Ce besoin de relations est souvent présent dans l'expérience d'abandon et les derniers instants sont aussi bien pour celui qui part que celui qui reste les moments où l'on peut régler ses affaires. Souvenons-nous de ce qui se passe au Golgotha. Jésus confie le disciple qu'il aimait à sa mère. Le disciple est re-suscité quand Il le donna à sa mère; Marie (la mère de Jésus) devient vivante quand elle reçoit son fils comme un cadeau du ciel. Dieu différencie cette mère de son fils, la relation reçoit son fils comme cadeau. "Jésus vit sa mère et, auprès d'elle, le disciple qu'Il aimait. Il dit à sa mère: "Voici ton fils, mère." 
Puis Il dit au disciple: "Voici ta mère." Et dès ce moment, le disciple la prit chez lui » (Jn 19, 26). Il est rassurant pour celui qui meurt de savoir que ses liens vont durer. Comment traverser la perte de l'amour? «Demeurez en moi comme moi...» Demeurez fidèles à ce moi qui est en vous comme je demeure en vous quand vous êtes atterrés par le deuil. Demeurez dans ce moi de la manière dont Dieu demeure en vous. Ne vous coupez pas de vous-même. Dans ce moi écorché vif se tient Dieu.

Il faut, dans le deuil, prendre soin de soi, c'est-à-dire de ce moi où se tient le divin. Le journal intime peut permettre de rester attentif à ce qui se passe en soi. Pensons au fils prodigue: père, perte, mort. Autant dire que le lien était détruit. Le lien le plus solide, c'est celui qui est passé par l'acceptation au moins potentielle de la perte du lien. C'est un apprentissage de vivre la perte du lien, l'apprentissage de l'amour inconditionnel. Mon lien d'amour a intégré la perte d'avance. Le lien le plus solide passe par l'acceptation de la perte. Le père voit son fils avec d'autres yeux. "Souffrir sans » ouvre la porte à «souffrir avec».

Le processus de deuil est à la fois psychologique et spirituel, ces deux aspects sont complémentaires et, dans la tradition chrétienne, le travail de deuil est fécondé par l'Esprit saint. Il faut prendre le temps de vivre le Vendredi saint même si on sait qu'il y aura Pâques. La personne qui le vit a l'impression que ça ne va jamais finir. Elle ne voit pas comment elle va pouvoir revivre de ses cendres. En visitant le négatif avec accompagnement, c'est à ce moment-là que vient la lumière. On accompagne l'autre jusque là où l'on est allé soi-même. Il y a toujours une issue: la Vie. "Jésus le regarda et l'aima.» Laissons Dieu être Dieu.

Les grands défis qui attendent la personne éprouvée par la mort d'un proche ou qui vit une autre perte sont les suivants: vaincre ses résistances au travail de deuil, intégrer l'in-intégrable et re-définir son image, pour enfin assurer la pérennité du lien. Les messages de l'Écriture et une oreille compatissante lui seront d'un précieux secours.

Les réflexions de Lytta Basset sur ces différents thèmes sont constamment appuyées sur les Écritures et sur des expériences spirituelles personnelles. Elle s'appuie également sur d'autres sources connues dans le domaine des études sur la mort: KüblerRoss, Bacqué et Hanus, Freud, Fauré, Ariès. On peut, sans contredit, contester cette nouvelle lecture des Écritures mais le croyant chrétien peut y puiser un message d'espoir quant au deuil, à la mort et à l'au-delà. La démarche proposée par Lytta Basset a le mérite de nous amener à pratiquer une brèche, à créer un espace nouveau pour une interprétation et un entendement non moins nouveaux des Écritures. Nous ne pourrons jamais plus lire de la même façon les passages cités ici.

\section{BIBLIOGRAPHIE}

ARIÈS, P. (1975). Essais sur l'histoire de la mort en Occident du Moyen-Âge à nos jours, Paris, Seuil.

BACQUÉ, M.-F. et M. HANUS (2001). Le deuil, Paris, Presses universitaires de France, coll. «Que sais-je?», n 3558.

COSTECALDE, C.-B. (dir.) (2000). Le Nouveau Testament en français courant, Strasbourg, Signe.

FAURÉ, $\mathrm{D}^{\mathrm{r}} \mathrm{C}$. (1995). Vivre le deuil au jour le jour, la perte d'une personne proche, Paris, Albin Michel.

FREUD, S. (1917). Mourning and melancholia, Standard edition, vol. 14, Londres, Hogarth Press.

KÜBLER-ROSS, E. (2005). La mort est un nouveau soleil, Monaco, Alphée.

LA BIBLE, nouvelle traduction, (2001). Paris, Bayard.

ÉCOLE BIBLIQUE DE JÉRUSALEM. (1995). La Bible de Jérusalem en gros caractères, Paris, Cerf.

\section{Note}

1. Les 23, 24 et 25 septembre 2005, à l'Institut de pastorale des Dominicains à Montréal, se donnait un cours intitulé: Deuil et spiritualité, donné par Lytta Basset, et dont le descripteur était le suivant:

Grand deuil ou «petits» deuils, la vie humaine est constamment confrontée à la perte et à la mort. S'il y a des deuils dont on n'arrive pas à sortir, par exemple parce qu'on se sentirait coupable d'être heureux et vivant, les passages déchirants de l'existence sont pourtant très souvent des hauts lieux spirituels. Mais à quelles conditions?

Ce cours, tel qu'il est décrit, propose une exploration de la réalité de la perte dans l'existence humaine. Qu'il s'agisse de travailler sa propre expérience ou d'apprendre à en accompagner d'autres dans la traversée de la mort, il sera utile de réfléchir sur, par exemple, les résistances au travail de deuil, l'intégration de l'inintégrable, l'incontournable redéfinition de l'image de soi, les indices d'une force de résurrection, l'avenir du lien.

Cet article n'a pas l'intention ni la prétention de reconstituer le cours ni d'en formuler une critique serrée sur le plan du contenu et de la forme. Il veut plutôt souligner les éléments que nous avons retenus, qui ont alimenté notre réflexion jusqu'à maintenant. 